\title{
Estertores en la clase de educación (física): Desmarcaciones con una pedagorgía
}

Rales in the (Physical) eEducation class: Standing out of one pedagorgy

\section{Sebastian Adolfo Trueba}

Facultad de Humanidades, Universidad Nacional de Mar

del Plata, Argentina

sebastiantrueba@gmail.com

\section{Francisco Ramallo}

Facultad de Humanidades, Universidad Nacional de Mar

del Plata. CONICET, Argentina

franarg@hotmail.com

\section{Resumen:}

La clase de educación física, en la normalizada y colonizada escolarización, condensó el ahogo de las normas del binarismo sexo-genérico. Desmarcados y en la necrocidad de la celebración de una pedagogía heterosexual, en este texto valorizamos una articulación de experiencias de investigación, extensión y docencia que respiran a partir de un modo de nombrar sofocante para la intelectualizada pedagogía crítica. Como pequeños ruidos y distracciones burbujeantes del fracaso de la educación (física), los hallazgos inmediatos movilizan una trama indivisible entre tres comunidades en común: el Grupo de Extensión Pedagorgía, la cátedra Teoría de la Educación y el Grupo de Investigación en Educación y Estudios Culturales (GIEEC) de la Facultad de Humanidades de la Universidad Nacional de Mar del Plata. Aludir a la metáfora de la respiración con los estertores kuir es un gesto que actúa, para nosotros respetuoso e irreverente, a favor de la autorización de una narrativa disidente para las teorías de la educación. Devenidas en orgías de la educación, desmarcamos desde ellas un horizonte que en el aquí y el ahora respira como kuir, en el entonces como queer y en el allí como cuir.

Palabras claVe: Binario, Educación física, Investigación narrativa, Pedagogía, Teoría queer.

\section{Abstract:}

The physical education class, in the normalized and colonized schooling, condensed the drowning of the gender-generic binarism norms. Standing out and in the necrocity of the celebration of a heterosexual pedagogy, in this text, we value an articulation of research, community-outreach and teaching experiences that breathe from a suffocating way of naming for the intellectualized critical pedagogy. Assmall noises and bubbling distractions from the failure of (Physical) Education, the immediate findings mobilize an indivisible plot between three communities in common: the Community-outreach Group called "Pedagorgy", the chair of Education Theory and the Research Group on Education and Cultural Studies (GIEEC) of the Faculty of Humanities of the National University of Mar del Plata. Alluding to the metaphor of breathing with Kuir rales is a gesture that acts, from our respectful and irreverent viewpoint, in favor of the authorization of a dissident narrative for the education theories. Coming from education orgies, we draw from them a horizon that breathes like kuir in the here and now, in the here as queer and in the there as cuir.

KEYWORDS: Binary, Physical education, Narrative research, Pedagogy, Queer theory.

\section{INTRODUCCIÓN}

“Lo queer aún no ha llegado, es una idealidad” (Muñoz, 2020, p. 37).

Insinuar ${ }^{\mathrm{i}}$ la metáfora de la respiración es un gesto que actúa respetuoso e irreverente, a favor de la autorización de una narrativa disidente para las teorías de la educación. Devenidas en orgías de la educación, marcamos desde ellas un horizonte que en el aquí y el ahora respira como kuir, en el entonces como queer y en el allí como cuir. Dado que no sólo no somos queer sino que nunca lo seremos, valorizamos la marca que José 
Esteban Muñoz trazó como horizonte de su potencialidad. Digamos que el futuro es del dominio de lo kuir, porque es un modo estructurante de desear que permite ver y sentir más allá del encierro del necropolítico presente. Lo cuir permite sentir que hay algo que falta y su insistencia en la potencialidad no es simplemente un ser, sino un hacer por y para el futuro (Muñoz, 2020). ${ }^{1}$

Movilizamos aquí los hallazgos inmediatos que entraman las experiencias de tres espacios comunes entre nosotrxs: el Grupo de Investigación en Educación y Estudios Culturales (GIEEC) de la Facultad de Humanidades de la Universidad Nacional de Mar del Plata, el Grupo de Extensión PedagOrgía y la cátedra Teoría de la Educación. En tal sentido, el presente artículo se desprende de una investigación que se está llevando adelante en los profesorados de educación física de la ciudad de Mar del Plata y que aborda desde un enfoque narrativo y autobiográfico los estertores binarios en la formación de docentes de educación física. Quizás porque advertimos, como ejercicios, algunos indicadores que manifiestan la importancia y urgencia de articular investigaciones en la formación de docentes de esta área de la escolarización en particular.

Nuestra docencia en la cátedra Teoría de la Educación ${ }^{2}$, se interesa especialmente por las narrativas que compiten por definir el territorio de lo educativo y la educación docente-esta última nombrada así como tensión con la "formación docente" para (des)ocultar la posibilidad de problematizar su continuidad, inmanencia, impermanencia y fluidez en los procesos de (re)configuración de las identidades profesionales que acontecen un ámbito vital más extendido que el de la ficción universitaria. Allí nos interesa subvertir la noción moderno/colonial de teoría/s que aun pugnan por instituir el campo pedagógico, desde un giro erótico que indagan las (in)visibilidades en el estudio de los cuerpos, los afectos, los deseos, el porno y la educación sexual en la composición del campo pedagógico en Argentina. La pedagogía narrativa, es invocada como modo de movilizar registros autobiográficos útiles para descomponer el disciplinamiento de su cientificidad.

La restauración de una erótica comunitaria en una pedagogía de la vivo es el movimiento no sólo intelectual sino también corporal y afectivo del que deviene el Grupo de Extensión Pedagorgía. ${ }^{3}$ Con metodologías transviadas y desgeneradas, reconocemos que a pesar de que las formas heteropatriarcales afectan profundamente los espacios educativos que habitamos, lo que llamamos pedagogía kuir y especialmente nuestra reincripción-Pedagorgía citrica- moviliza prácticas y discursos que intervienen en los efectos de su normalidad. Acompañados por la experiencia de aprendizaje en educación viva y adulta que comparte el Canto del Fuego ${ }^{4}$, afirmamos que podemos huir de las heridas que están en nosotres y deseamos intervenir en las desigualdades existentes para acceder a dinámicas de aprendizaje comunitario en el espacio público.

El lazo erótico y comunitario que descomponemos con El Canto del Fuego, en la invitación a activar una experiencia de educación viva para adultes, enraíza el autoconocimiento, la experiencia sensorial, la conciencia corporal y la expansión de la afectividad. De allí radica el nombre de este grupo, a la vez que propone disputar la representación de los sentidos extractivistas asociados a la erótica de lo grupal y que colocan a los saberes de la educación en un primer plano. Reconociendo de este modo, que nuestros modos de conocer, ser y saber están profundamente afectados por nuestras corporalidades y sexualidades. En intermedio de lo porno que recoge el famoso lema "sexo grupal derriba el capital", aquí nos interesa resaltar el valor de componer una comunidad de aprendizaje en la que la liberación del conocimiento no sea sólo intelectual sino también corporal y afectiva (Hooks, 2016 y 2019). Desde la restauración de nuestras capacidades afectadas y afectivizantes, el placer deviene en una oportunidad para subjetivar nuestros deseos y no sólo ser cuerpos al servicio de la objetivación del régimen farmacopornográfico (Preciado, 2011). Es por ello que el sufijo orgía no debe leerse en los términos sexuales de la normalidad hétero (Britzman, 2016a), sino más bien en la potencia de cuerpos pulsantes que se reconocen a sí mismos en la expansión colectiva de sus deseos.

En síntesis, argumentamos aquí que la clase de educación física, en la escolarización moderna, condensó el ahogo de las normas del binarismo sexo-genérico. Desmarcados en la necrocidad de la celebración de una 
pedagogía heterosexual, en este texto valorizamos una experiencia de investigación, extensión y docencia que respira a partir de un modo de nombrar sofocante para la clásica pedagogía crítica: pedagorgía cítrica.

\section{Orgías PARA LA EDUCACIÓN (FÍSICA)}

Frente al diagnóstico erotizante del peligro en la imaginación (Muñoz, 2020), las orgías de la educación nombran al devenir kuir que fuimos habitando en una profundización de dos investigaciones espejadas (Ramallo, 2019; Trueba, 2019a; Trueba, 2019b; Trueba, s/p). Tramar una investigación desde los institutos de formación docente de la ciudad de Mar del Plata, eclipsa algunas de las problemáticas evidenciadas tanto en esta exploración cualitativa como en indagaciones cuantitativas y estadísticas. La "Encuesta del clima escolar dirigida a jóvenes LGBT" realizada en la Argentina en el año 2016, ofrece singulares datos para los deslices kuir (Ramallo y Gómez, 2019). La conversación entre ambos registros apostó a la inestabilidad -o ambigüedad- de un lugar o un momento que actúa como un flujo descompuesto y como una desorganización del tiempo y la lectura hetero lineal.

La intertextualidad de la mencionada encuesta destaca tres preguntas que son muy elocuentes en referencia a los géneros y las sexualidades en las clases escolarizadas de la educación física. Atentos a su lectura no recta -o hetero- (Britzman, 2016a), anteponemos tres interrogantes que nos regaló Susanne Luhmann (2018): ¿Podemos resistir el deseo de autoridad y de conocimiento estable, haciendo estallar las certezas sobre lo que se sabe y sobre cómo llegamos a saberlo?,¿Podemos desplazar el interés por las metodologías y técnicas para poner el foco en los múltiples e inasibles modos en que lxs estudiantes son perturbadxs por el conocimiento? y ¿Podemos soportar que nuestrxs estudiantes no soporten conocer lo que pretendemos enseñarles?. La pedagogía como una cosa bastante cuir, en una propuesta postidentitaria sobre la afectación de sus conocimientos que resalta estas tres exploraciones conjuntas -en nuestra simplificación- está atenta a marcar que: "Les preguntamos a los estudiantes LGBT si había espacios particulares en la escuela que evitaran específicamente por sentirse inseguros o incómodos (...), los estudiantes LGBT generalmente evitaban las clases de gimnasia o educación física (...)" (AA.VV, 2016: 12).

En nuestra lectura lo más revelador desde este régimen de representación es que esta respuesta computó más del $43 \%$ de lxs encuestadxs. Y más aún su repetición se entrama con otras en el mismo sentido.

Para entender si ciertos tipos de personal de la escuela era más propensos a ser visto como un apoyo, preguntamos a los estudiantes LGBT cuán cómodo se sentirían hablando a solas con algunos miembros del personal de la escuela sobre cuestiones relacionadas a lo LGBT. (...) los estudiantes informaron que se sentirían más cómodos hablando con profesionales de la salud mental en las escuelas (consejero, trabajador social, psicopedagogo o psicólogo de la Escuela): un 63,7\% dijo que se siente un poco o muy cómodo hablando de temas LGBT con este personal (...). Aproximadamente la mitad de los estudiantes LGBT dijo que se sentirían algo o muy cómodos hablando con "profesor / as" y "preceptor / as." Menos estudiantes en nuestra encuesta dijeron que se sentirían cómodos al hablar a solas con entrenadores deportivos o con el profesor de educación física y con el personal de limpieza o mantenimiento (AA.VV, 2016: 38).

Asimismo, solo el 32,2\% de lxs jóvenes plantearon comodidad al hablar de estos temas con lxs profesorxs de educación física, lo que podría leerse también como que casi el $70 \%$ no se sienten cómodxs.

Se pidió a los estudiantes en nuestra encuesta si les habían dado representaciones de las personas LGBT, su historia, o eventos en las clases en la escuela, y más de la mitad $(54,5 \%)$ de los encuestados dijeron que en sus clases no se tratan estos temas (véase la Figura 2.6). De los estudiantes que dijeron que sí se les había enseñado sobre temas LGBT, aproximadamente una cuarta parte (26.1\%) dijo que los temas LGBT se habían discutido sólo en una manera positiva en una o más de sus clases, y un $3.2 \%$ adicional de los estudiantes informó que estos temas se debatieron tanto en formas positivas como negativas. Entre los estudiantes a los que les enseñaron cosas positivas sobre temas LGBT en clase, "Formación Ética y Ciudadana" y "salud", fueron las clases que más se mencionan como incluyentes de estos temas (AA.VV, 2016: 38).

Sumado a ello, solo el 3,5\% de lxs encuestadxs manifestó haber vivido abordajes de estos temas en las clases de educación física. Lo más preocupante es que los diseños curriculares de las diferentes jurisdicciones 
plantean el abordaje de estos temas. Por ejemplo, si tomamos el diseño curricular de $4^{\circ}$ año de la escuela secundaria de la provincia de Buenos Aires encontraremos que entre las estrategias de enseñanza sugeridas se expresa lo siguiente: "Acordar propuestas que surjan de la confluencia de materias diferentes para el tratamiento articulado de temáticas como sexualidad, prevención de adicciones, alimentación, higiene y violencia, entre otros" (DGCyE, 2010: 32)

En la sección contenidos, entre otros, se prescriben los siguientes:

- El desarrollo en grupo de proyectos personalizados de actividad motriz y su relación con la autoestima y el respeto a los otros. (DGCyE, 2010: 15)

- La imagen corporal y el impacto de los modelos mediáticos y de la mirada de los otros en su constitución. Su análisis crítico. (DGCyE, 2010:15)

- La organización y desarrollo de actividades motrices compartidas con cuidado y respeto corporal entre los géneros. (DGCyE, 2010:15)

- Tareas de concientización corporal. La sensopercepción del propio cuerpo. Su relación con la autoidentificación y la comprensión de la propia corporeidad. (DGCyE, 2010:16)

Si bien la encuesta aporta indicios muy sugerentes respecto a que estos contenidos no se desarrollarían en muchas clases de educación física, hay que aclarar que en cada jurisdicción los contenidos y las estrategias presentan algunas diferencias. En un estudio exploratorio realizado el año 2019 en un profesorado de educación primaria de la ciudad de Mar del Plata surgieron indicios que refuerzan las ideas sugeridas en la encuesta planteada anteriormente. Se les solicitó a veinte y dos docentes en formación de tercer año del profesorado de educación primaria que narraran su mejor experiencia en una clase de educación física. Solo tres respondieron que tenían experiencias positivas que contar, mientras que las diecinueve restantes no pudo hallar nada positivo en ese momento. De hecho, hubo cinco estudiantes que expresaron frases como: "odio la educación física", "no puedo encontrar nada positivo en esa materia" o "pasar por esa materia fue traumático". El ejercicio implicaba insistir en recuperar las mejores experiencias y de a poco comenzaron a aparecer, sin embargo, los pocos momentos destacados solían estar relacionados a actividades en las que no intervenían directamente lxs profesorxs del área. Por ejemplo, cuando organizaban la preparación de un esquema gimnástico entre las compañeras o cuando se organizaba alguna jornada de juegos y prácticas deportivas mixtas.

Al consultar a otrxs docentes que dictan la materia "Educación física escolar" en esa institución, todxs me confirmaron que esos relatos son muy habituales. Ante ello nos preguntamos: ¿no sería necesario intervenir en las instituciones formadoras para comprender por qué continúan apareciendo narrativas con estos sentidos excluyentes, heteropatriarcales, binarios, lineales y discriminadores? ¿Cómo se pueden interrumpir estas lógicas? ¿somos conscientes lxs docentes de esta situación? ¿luchamos, resistimos o reproducimos el dualismo en la formación docente? Por otro lado, ¿cómo identificar estertores de estas políticas? ¿qué posibles intervenciones son posibles en este contexto?

Esta interrogación busca la activación de una pasividad radical (Halberstam, 2011), es decir, un modo disidente de reinvidicar el arte del fracaso queer y la continuidad sistémica que el éxito promueve. Además coincidimos con Susanne Luhmann (2018) en que una pedagogía cuir no es heroica, en el sentido que no se posiciona como baluarte contra la opresión y no reivindica un plano superior de subversión. A pesar de ello, nuestra expectativa no renuncia a que este texto aliente prácticas de lecturas atentas a los riesgos de la normalización (Luhmann, 2018, 62). Diremos, entonces, que intenta generar el empujón inicial para comenzar un posible abordaje de la cuestión y en ese sentido es que nos sometemos a la desestabilización cuir y provocamos lecturas no rectas -o hétero- (Britzman, 2016a) de la educación (física). 


\section{DESMARCACIONES CON LA EDUCACIÓN (FÍSICA)}

La disidencia no es diferencia, no somos otros sino que somos nosotrxs. El asumir la nostredad ante del fracaso del mundo, nos convierte en sujetos de nuestros propios deseos como radicalidad del voyeurismo científico. Para activar el develamiento del carácter ficcional del conocimiento de las disciplinas científicas en una reciprocidad, colectividad y plasticidad en la que lo privado es público, descomponemos el lugar de derecho y de privilegio del ser docente (Flores, 2017). Nuestra enunciación más que como "guardianes de la democracia" - como nos diría la pedagogía crítica, que acompaña a la profesionalización e institucionalización de una sistemática y divina pedagogía (crítica)-se descompone al nombrar los sexos, los afectos y los cuerpos desde un habitar comunitario de la intimidad.

También Mary Bryson y Suzanne de Castell (2018, p. 128) rechazaron el ocultar de las sexualidades que promueven la pluralista y mandataria institucionalización de la pedagogía crítica, en el reconocimiento de una típica prescripción educativa común que aborda los "problemas" de las “identidades" de la disidencia sexual en algún tipo de exhortación pluralista al "diálogo a través de las diferencias". En contraposición aparece la intimidad y del mismo modo que se ha desacreditado el "sexo seguro" no puede haber una "pedagogía segura". En definitiva, una pedagogía segura es considerar las posibilidades que ofrece la noción de una "ética del consumo y de la producción" para reformular las prácticas pedagógicas o pretender saber más acerca de gays, bi, trans o lesbianas para pensar en un "cruce de fronteras" asimilacionista. La institucionalización de la pedagogía crítica parece tener mucho más en común con un tipo de "turismo intelectual" colonizante y sus consiguientes estrategias de despojo y apropiación cultural masiva, que con cualquier intento de travesía reflexiva hacia las desconocidas y poco analizadas "diferencias y opresiones interiores" (Bryson y de Castell, 2018, p. 131). El valor de una pedagogía cuir está en el cuirizar de sus técnicas y en garabatear grafitis sobre sus textos, colorear fuera del contorno para tomar deliberadamente el camino equivocado al ir a la escuela o ir en una dirección totalmente diferente de la establecida por un destino monológico (Bryson y de Castell, 2018, p. 129).

Entre el texto y la presencia, referiremos a una secuenciación de cinco desmarcaciones identificadas como binarismos que son especialmente recurrentes en la educación (física). Entre la representación y la acción, rebalsan su lugar de dato y hacen estallar la cotidianeidad de lugares comunes en sus prácticas disciplinares. Entre el contenido y la forma, presentamos cinco cápsulas no exhaustivas ni totales que desmarcan dicotomías que sostienen y que son sostenidas por las normas del binarismo sexo-genérico en la educación (física).

\section{Homosexualidad-heterosexualidad}

¿Qué campo tan particular la educación física! Somos miles de docentes, lo que supondría una amplia diversidad. A pesar de eso se sostiene una aparente homogeneidad, al punto tal que podemos jugar a identificar profes en cualquier lugar solo por reconocer nuestros rasgos comunes (prejuicios surgidos del sentido común) a saber: -los profes no son afeminados ni delicados, -mayoritariamente no se manifiestan homosexuales, valoran la hombría, la fuerza y el sacrificio, (ipongan huevo! ¡no sean maricones! ¡le pegás como una nena! Son expresiones comunes en nuestras clases) -hablan fuerte, -Las profesoras pueden ser machonas (poco femeninas), -en el caso de las mujeres se puede manifestar su orientación sexual disidente (siempre y cuando sea lesbianismo), -no son excesivamente delicadxs -ni hombres, ni mujeres- en el trato (aunque sí pueden ser cariñosxs), etc. Es llamativo que entre los hombres no se permita la delicadeza y feminidad, mientras que para las profesoras sí se acepte la masculinidad. De hecho conozco muchas docentes lesbianas y no conozco un solo docente que se declare homosexual. Pareciera ser una marca más que refuerza como positivas las huellas heteropatriarcales que suponen un estatus superior para lo masculino. 


\section{Cuerpo-mente}

Por momentos pareciera ser el gran dualismo de la educación (física) que aún no cuenta con fecha de caducidad. En realidad, deberíamos decir que se trata de uno de los grandes dualismos de la educación y del pensamiento, porque la escisión de educar la mente y habitar un cuerpo como algo exterior a nosotrxs, se manifiesta en el colectivo docente. Hace décadas que se propone desde documentos oficiales, los diseños curriculares y la mayoría de la bibliografía publicada, la ruptura del dualismo mente-cuerpo y a pesar de eso persiste y resiste a su muerte. Desterrando un posicionamiento de solapamiento en el que, desde un discurso aparentemente superador, se mantiene vivo al binarismo. Nos referimos a los "estudios del cuerpo", cuando se extienden a la idea de que no somos cuerpos y estamos analizándonos a nosotrxs, sino que se estudian cuerpos desde un posicionamiento objetivista que necesita/desea despersonificarlo. Como si pudiera escindirse el cuerpo de nosotrxs.

\section{Mujer-varón}

Un clásico de las clases de educación física es la división de actividades por sexo/género, en el que planteamos tareas diferentes para unas y para otros. Al realizar el estudio exploratorio previo a esta investigación, intentamos recuperar las mejores experiencias que docentes en formación de educación primaria habían vivido en su escolarización. Y lo que surgió de manera recurrente fue el recuerdo de esta división impuesta por lxs docentes; al tiempo que destacaron, como sus mejores experiencias, las posibilidades de realizar actividades mixtas sin intervenciones directas de sus profesorxs. ¿Podremos algún día plantear propuestas para nuestrxs alumnxs sin parapetarnos en esta dicotomías?

\section{Niñx-adulto}

Este binarismo suele ser el más invisibilizado, pero no es menor. De hecho, muchxs docentes se sienten mucho más cómodxs trabajando con algún rango etario en particular. Están quienes solo trabajan en nivel inicial o solo en secundaria por sentirse incómodxs en otros grupos. Sin embargo, cuando se minimizan problemáticas por ser cosas de niñxs, o cuando no se les da la posibilidad de participación en las decisiones por ser chicxs, estamos asistiendo a un refuerzo de este dualismo. El bulling, en cierta medida, se fomenta cuando lxs docentes no se ponen en el lugar de lxs pequeñxs y desisten en sus intentos de percibir la complejidad de su mirada. Todo binarismo plantea una distancia difícil de acercar. O se es adulto y por ende se sabe, o se es niñx, y por lo tanto se ignora.

\section{Teoría-práctica}

Esta es representada y reproducida por gran parte del colectivo docente, y no solo por lxs de educación física. Solemos escuchar que hay materias teóricas y otras que son prácticas. Las unas requieren pensar y las otras implican hacer. Se puede observar un cierto grado de incertidumbre cuando unx profesorx de educación física plantea una clase teórica. Y dicha incertidumbre pasa a rechazo cuando se plantea la evaluación de dicha clase. Lxs mismxs docentes menospreciamos la reflexión y la exégesis cuando no se trata de algo simplista y concretx. En cierta medida se puede inferir una relación con el binomio conocimientoignorancia, relacionando al conocimiento con la teoría y a la práctica con la ignorancia. Deborah Britzman (2016b) va un poco más allá y nos permite cerrar el bucle al plantear: 
El viejo dualismo binario de ignorancia y conocimiento no puede reconocer el hecho de que cualquier conocimiento ya contiene sus propias ignorancias. Si, por ejemplo, la gente joven, o lxs educadorxs son ignorantes acerca de la homosexualidad, podemos apostar que saben poco acerca de la heterosexualidad (p. 61).

Entonces ¿Puede haber práctica sin teoría o teoría que no refiera a la práctica? ¿podemos escindirnos al punto de hacer sin reflexionar o lo contrario? :por qué sostenemos el carácter binario del conocimiento? Prácticas débiles (no potentes) suelen estar imbuidas en marcos conceptuales superficiales (Maggio, 2016).

\section{ESTERTORES CON UNA PEDAGORGÍA}

La utopía cuir es una hoja de ruta que no busca la mera inclusión en un orden social corrupto y arruinado, ni tampoco rechaza la acción política colectiva de una comunidad de ciudadanos normativos. Para Muñoz (2020) el pensamiento hétero hace que pensemos en episodios cerrados y unívocos, sin embargo, en él hay un agite de múltiples fuerzas, sueños y utopías concretas no realizadas y confinadas a un todavía no. Es para ello que nos propone marcarse, rescatar marcas y huellas utópicas. La presencia del futuro en las marcas que restauramos, devienen en un desplazamiento entre el "aquí y ahora" y el "entonces y alli’" (Muñoz, 2020; 182). El tiempo exacto del realismo, que amarra el presente como espacio y como tiempo, pueda ser abandonado a favor de registrar la presencia del futuro en las huellas pretéritas. Frente al diagnóstico erotizante del peligro de la imaginación, apostamos a la inestabilidad -o ambigüedad- de un lugar o un momento que puede situarse tanto en el futuro como en el pasado, dado que la utopía queer actúa como un flujo descompuesto y como una desorganización del tiempo hetero lineal. Este encuentro apuesta, entonces, a registrar un pasado que aún no es pensable, posible o legible. Su propósito es hacer saltar el continuum de la historia, quizás en una futuridad antinormativa y desmarcada. Por eso, en este esfuerzo por nombrar nuestro entonces y allí, es que tomamos como el tamiz de las redes que descomponemos. Nuestra intención es justificar una lectura que restaure al placer por encima de las heridas de la objetivación, la normalidad y la colonialidad de la anacrónica y universal realidad. Con el desafío de compartir el reconocimiento de las posibilidades utópicas, que ancladas en la inmediatez y en la fluidez del paisaje social que habitamos (des)ocultan la descomposición de las clases de educación (física) en las que estamos inscriptxs.

Las disciplinas califican y descalifican, legitiman y deslegitiman, premian y castigan; se reproducen a sí mismas de forma estática para reprimir la disidencia. Jack Halberstam (2011), una pensadora trans del norte, caracterizó a esta época como benevolente para experimentar una transformación disciplinaria en las formas de conocimiento; dado que las disciplinas que se crearon hace cien años, para responder a las economías de mercado y la demanda de una experiencia limitada, ahora están perdiendo relevancia y son incapaces de responder al conocimiento del mundo real o a los intereses de sus estudiantes (Halberstam, 2011, p.19). Por tanto, cobra sentido su interrogante “¿realmente queremos apoyar los desgastados límites de nuestros intereses compartidos y de nuestros compromisos intelectuales, o podríamos más bien aprovechar esta oportunidad para repensar el proyecto del aprendizaje y del pensamiento? (Halberstam, 2011, p.19).

En tal sentido quisiéramos reforzar la idea de que la falsedad y el simplismo que implica el pensamiento binario tiene consecuencias indeseables no solo para quienes no cumplen con los estándares sociales de la cultura cis-hétero-normativa sino para todxs los expuestxs en este sistema. En la clase de educación física estudiantxs y profesorxs se exponen constantemente a la extrema agresividad de la heteronormatividad, quien no cumple padece al igual que quien debe forzarse a llegar a un cierto nivel de exigencia para no caer en el escarnio y la burla. Aquí nos preguntamos de manera similar a como lo hizo Facundo Saxe (2018) respecto de los modos de descomponer el conocimiento científico: ¿por qué no podemos construir modalidades de pensar la clase de educación física desde el placer? $\mathrm{O}$ ¿por qué nos cuesta tanto asociar la educación física con el placer?

La educación física construyó una historia que la ha convertido en una disciplina anticuir -si existiera la palabra-. Sin embargo curiosidades, experiencias e inquietudes comienzan a movilizar a colectivos docentes 
en pos de una desestabilización de la disciplina que posibilite la vivencia de alternativas al dañado cuerpo heteronormativo. Entre las carencias podemos mencionar las actitudes destacadas a lo largo del trabajo y que cada vez son más visibles entre el profesorado, como se destaca en un estudio (Piedra de la Cuadra, et al, 2013) realizado entre docentes en formación de educación física españolxs. En términos generales sabemos que el alumnado sigue percibiendo comportamientos homofóbicos y heterosexistas en las clases de educación (física). Disminuyendo las diferencias culturales, al comparar con estudios en otros países que coinciden con los resultados obtenidos tanto desde la indagación cuantitativa referenciada como desde nuestra exploración cualitativa, los datos parecen apuntar hacia la idea de que los cambios legislativos producidos en las sociedades desarrolladas en los últimos años en materia de igualdad, no están siendo acompañados por un cambio de mentalidad entre los individuos en materia de orientación sexual por la continuidad de un necrosado curriculum heterosexual (Piedra de la Cuadra, Rembrandt Rodriguez Sánchez, Ríes, Ramírez Macías, 2013, p. 336). Tal como se remarca fuertemente en los trabajos que lleva adelante el Grupo Actividad Física, Educación y Sociedad (AFES) de la Universidad de Valencia, en relación al conocimiento y la sensibilización de las problemáticas de la población trans-queer en la educación física (Fuentes-Miguel, J; Pereira-García, S; López-Cañada, E; Pérez-Samaniego, V. \& Devís-Devís, J., 2018; Devís-Devís, Pereira-García, FuentesMiguel, López-Cañada, \& Pérez-Samaniego, V, 2018).

Estas insuficiencias no lo son todo. En nuestros espacios de docencia, investigación y extensión valorizamos y potenciamos la posibilidad de imaginar y vivir clases de educación (física) desmarcándonos de las heridas del daño de la heteronormatividad. Del mismo modo que posicionamos a lxs docentes de educación física en un lugar de privilegio para componer una pedagogía corporal, afectiva, sexual y no sólo intelectual. Lejos de delimitar los estertores de este sistema, en estas páginas invitamos a escuchar sus horizontes, recogiendo los deslices de ciertos binarismos, que como simplificaciones terminan naturalizando la animosidad a la disidencia (sexual). Entre la encuesta de clima escolar para población LGBT y las históricas experiencias frustrantes de muchxs niñxs y jóvenes que transitan las clases de educación (física), descomponemos una pedagogía (cuir) que no promete ni trasgrede autoarrogantemente, sino que por el contrario respira al ritmo de la vida -no solo humana-.

En la interdependencia manifestada por el aislamiento social preventivo y obligatorio (ASPO) en el que nos encontramos la mayoría de las sociedades del mundo a partir de la pandemia del COVID-19, desnudamos un denso territorio a la espera de modos posibles de su compresión y más aún de la composición de marcas para futuros no normativos. La urgencia de la violencia sobre los cuerpos desjerarquizados por el capitalismo, el colonialismo, la modernidad y el patriarcado, se volvió desgarradoramente obscena con el encierro (Maffía, 2020; De Sousa Santos, 2020: 35), a pesar de ello en las hoy virtualizadas clases de educación (física) abrazamos una enorme oportunidad.

\section{Referencias bibliográficas}

AA. VV (2016). Encuesta del clima escolar dirigida a jóvenes LGBT. 100\% Diversidad y derechos 7(9), Pp. 13-34.

Britzman, D (2016a) “¿Hay una pedagogía queer? O, no leas tan recto. Revista de Educación de la Facultad de Humanidades No9, Año 7. Pp. 13-34.

Britzman, D (2016b). ¿Qué es esa cosa llamada amor?, en Pedagogias transgresoras. Córdoba, Bocavulvaria ediciones.

BRYSON, M y De Castell, S (2018) “Pedagogía cuir: La práctica hace la im/perfección” en Pedagogías transgresoras II. Santa Fé, Bocavulvaria.

De Sousa Santos, B. (2020). La cruel pedagogía del virus. Buenos Aires, CLACSO.

Devís-Devís, J; Pereira-García, S; Fuentes-Miguel, J; López-Cañada, E. Y Pérez-Samaniego, V. (2018). Opening up to trans persons in Physical Education-Sport Tertiary Education: two case studies of recognition in queer pedagogy. Physical Education and Sport Pedagogy, Londres. Routledge. 
Dirección General de Cultura y Educación de la provincia de Buenos Aires (2010).Diseño Curricularpara la Educación Secundaria ciclo superior / ES4: Educación Física. La Plata, Provincia de Buenos Aires.

Flores, V. (2017). Interruqciones: Ensayos de poética activista, Escritura, política, pedagogía. Córdoba, Asentamiento Fernseh.

Fuentes-Miguel, J; Pereira-García, S; López-Cañada, E; Pérez-Samaniego, V. Y Devís-Devís, J. (2018). Más allá del binario sexo/género: una propuesta pedagógica trans-queer en Educación Física. En Eloísa Lorente Catalán y Daniel Martos I García (comps.) Educación Física y pedagogía crítica propuestas para la transformación personal y social.Universidad de Valencia/Universidad de Lleida.

Halberstam, J (2011) El arte queer del fracaso. Madrid, Egales.

Hooks, B. (2016). Eros, erotismo y proceso pedagógico, en Pedagogías transgresoras. Córdoba, Bocavulvaria ediciones.

Hooks, B (2019). Ensinando a transgredir: a educação como prática da liberdade. Sao Pablo, Editora WMF Martins Fontes.

Luhmann, S (2018) ¿Cuirizar/cuestionar la pedagogía? O, la pedagogía es una cosa bastante cuir. En Herczeg, G y Adelstein, G (traductoras). Pedagogías transgresoras. Santa Fé, Bocavulvaria.

Maffía, D. (2020). La violencia de género ¿la otra pandemia?, en A. Grimson (coord.): El futuro después del COVID-19, Jefatura de Gabinete, Argentina.

Maggio, M. (2016). Enriquecer la enseñanza. Los ambientes con alta disposición tecnológica como oportunidad. Bs. As. Paidos.

Muñoz, Je (2020) Utopía queer: El entonces y alli de la futuridad antinormativa. Buenos Aires, Caja Negra.

Piedra de la Cuadra, J; Rembrandt Rodriguez Sánchez, A; Ríes, F; Ramírez Macías, G. (2013). Homofobia, heterosexismo y educación física: percepciones del alumnado, en Profesorado. Revista de curriculum y formación del profesorado. Vol.17, ํ⒈ Universidad de Granada.

Preciado, Pb (2011) Manifiesto contrasexual. Barcelona, Anagrama.

Ramallo, F (2019) Paulo Freire con glitter y pañuelo verde: Notas cuir para educadores. Série-Estudos, Campo Grande, MS, v. 24, n. 52, p. 101-122, set./dez. http://www.serie-estudos.ucdb.br/index.php/serie-estudos/article/view /1336/pdf

Ramallo, F. y Gómez, J. (2019). “Deslices kuir: una presentación para no leer tan recto”, en Revista de Educación, Año $X, \mathrm{~N}^{\circ} 18$, pp. 13-18.

Saxe, F. (2018). La trampa mortal: derivas maricas de la disidencia sexual en la producción de conocimiento científico al recuerdo infantil de un beso, en Etcétera. Revista del Área de Ciencias Sociales del CIFFyH, N.3. Córdoba: UNC. Recuperado de https://revistas.unc.edu.ar/index.php/etcetera/article/view/22591

Trueba, S. (2019a).Profesores memorablespara los docentes formadores de losprofesorados de Educación Física de la ciudad de Mar del Plata. Tesis del Doctorado en Humanidades y Artes con mención en Ciencias de la Educación. UNR.

Trueba, S. (2019b). Educación física cuir ¿̇un largo camino por recorrer?, en Revista de Educación, Año X, №18, pp. 261-273.

Trueba, S. (s/p). ;Basta de poner el cuerpo: seamos cuerpo! Reflexiones acerca de: corporeidad, cuerpo y si mismx. Mimeo

\section{Notas}

i Datos de los autores: Trueba es Docente e investigador del Departamento de Ciencias de la Educación de la Facultad de Humanidades (UNMdP), miembro del Grupo de Investigación en Educación y Estudios Culturales del Centro de Investigaciones Multidisciplinares en Educación (CIMED). Profesor de Educación Física (ISFD No84), Licenciado en Educación Física (UNL) y Doctor en Humanidades y Artes con mención en Ciencias de la Educación (UNR). Ramallo es Docente e investigador del Departamento de Ciencias de la Educación de la Facultad de Humanidades (UNMdP) y Becario Posdoctoral de CONICET, miembro del Grupo de Investigación en Educación y Estudios Culturales del Centro de Investigaciones Multidisciplinares en Educación (CIMED). Doctor en Humanidades y Artes con mención en Ciencias de la Educación (UNR). 
1 Este juego de palabras entre queer, cuir y kuir, abraza los deslices kuir (Ramallo y Gómez, 2019) como una forma de no leer tan recto -o tan hétero diría Briztman (2016)-. Es decir, la utilización indistinta de diferentes formas de referenciarla. Dirían Ramallo y Gómez (2019, p. 13): "Manoteamos el lenguaje “inclusivo” para llamar a lo kuir. Lo agarramos para no normativizar su reapropiación. Como gesto performático apelamos a su escritura fonética en castellano (cuir), lo escribimos con k para elogiar su descomposición y para aplaudir al próximo gobierno en Argentina (kuir), abrazamos el origen y la historicidad del vocablo en inglés porque lxs gringuxs también suelen hacernos felices (queer). Y jugamos con colegas a cuirizarnos (huir). Ese es nuestro desliz. Nos olvidamos del preservativo".

2 El equipo docente de la Cátedra Teoría de la Educación de la Licenciatura en Ciencias de la Educación de la Facultad de Humanidades de la Universidad Nacional de Mar del Plata está conformado por María Marta Yedaide, Francisco Ramallo, Sebastián Trueba, Ileana Correa y Ornela Barone Zallocco.

3 Los autores de este texto dirigimos el Proyecto de Extensión "Cuir en educadores: Talleres, performances y jornadas de educación viva para generar materiales didácticos disidentes" (2020-2021) radicado en el Grupo de Extensión PedagOrgía Facultad de Humanidades y financiado por la Secretaría de Extensión Universitaria de la Universidad Nacional de Mar del Plata.

4 El Canto del Fuego es una comunidad de aprendizaje de educación viva y libre de la ciudad de Mar del Plata con la cual cohabitamos nuestro grupo de extensión. Su cercanía nos permite experimentar en el seno de la Universidad Nacional de Mar del Plata una experiencia de educación viva y libre para adultxs. Las incomodidades y desorientaciones, son dos factores que articulan el sentir, hacer y pensar que erotiza en este equipo el compromiso de abrir espacios de conocimientos autogestionados para la comunidad. Y nos provoca a desencajonar saberes y despeinar gominas en la díscola extrañeza del encanto. 